\title{
Fabrication of a Silicon-Based Superfluid Oscillator
}

\author{
Keith Schwab, J. Steinhauer, J. C. Davis, and Richard E. Packard
}

\begin{abstract}
We have constructed an integrated superfluid oscillator using various silicon processing techniques, including micromachining and electron beam lithography. This device has the advantage of a very small internal volume $\left(0.72 \mathrm{~mm}^{3}\right)$. This makes it insensitive to spurious external acoustic noise which has limited the performance of larger experiments. We have tested the performance of this device in two configurations, one with a single micro-aperture and another with an additional fine tube. Both configurations demonstrate macroscopic quantum phenomena in superfluid ${ }^{4} \mathbf{H e}$ at low temperatures $(0.25 \mathbf{K}<T<2.2 \mathbf{K})$ and have been used to study these effects in detail. [169]
\end{abstract}

\section{INTRODUCTION}

A PPROXIMATELY ten years ago, Avenel and Varoquaux first demonstrated that individual quantized phase slip events could be detected using a superfluid Helmholtz oscillator [1]. These phase slips, which occur in small flow apertures, are detected by observing abrupt drops in the oscillator's amplitude and correspond to an oscillator energy change of $\sim 1 \mathrm{eV}$. The amplitude drops are quantized dissipation events, caused by the nucleation and subsequent motion of a single quantized vortex. The phase slips constitute the primary channel for the dissipation of superflow in small apertures and are responsible for the so-called "critical velocity." For velocities below this critical velocity, the fluid moves essentially as an ideal, dissipationless fluid. For velocities above this critical velocity, however, the rate of phase slip vortex nucleation and consequent dissipation becomes appreciable. This superfluid oscillator technique has created a new opportunity to explore some interesting problems in superfluid physics including questions about the origin of quantized vortices [2], their role in the critical velocity, and the role of macroscopic phase coherence in absolute rotation [3].

An important technical problem with these superfluid oscillators is that acoustic noise in the laboratory environment, coupled to the oscillator by vibrations, easily triggers phase slip events. This often limits the precision of measuring the phase slip critical velocity (because the spurious velocities are unknown) and can completely obscure phase coherence phenomena such as the two-hole staircase pattern. It was conjectured that acoustical modes in the superfluid sample were being driven by mechanical vibration of the entire apparatus. These sound fields and associated velocity fields in the fluid could be responsible for the wide distribution of

Manuscript received August 22, 1995; revised May 16, 1996. Subject Editor, S. D. Senturia. This work was supported by the Office of Naval Research and the National Science Foundation.

The authors are with the Physics Department, University of California, Berkeley, CA 94720 USA.

Publisher Item Identifier S 1057-7157(96)06837-0.

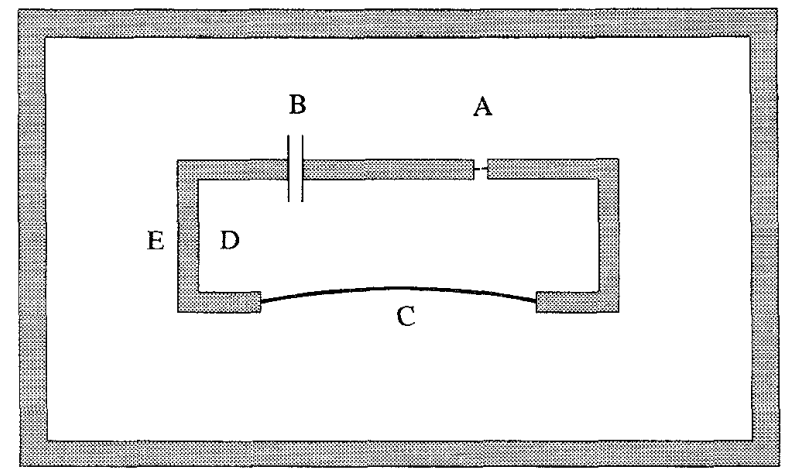

Fig. 1. The generic Helmholtz oscillator: (a) micro-aperture, (b) parallel path, fine tube, (c) flexible diaphragm, (d) helium filled interior of device, and (e) helium filled superfluid reservoir.

phase slip critical velocities and the lack of staircase pattern for these larger cells.

One approach to resolve this hypothesis and to possibly improve oscillator performance is to construct elaborate vibration and acoustic isolation. Such an approach can be expensive and space consuming [4]. Another approach is to make the entire apparatus so small that the lowest lying acoustical modes of the superfluid helium lie far above any ambient noise in the laboratory. We have elected the latter route and report here the construction techniques and performance characteristics of a silicon miniaturized Helmholtz oscillator. ${ }^{1}$

\section{THE GENERIC OSCILLATOR}

Geometrically, our oscillator is very similar to the design reported by Avenel and Varoquaux; however, in construction technique and detail, it is substantially different. The basic geometry of such a Helmholtz oscillator is shown in Fig. 1. A superfluid-filled box nests within a larger superfluid reservoir. The inner box is connected to the surrounding volume by a submicrometer sized aperture; in our case this is constructed by electron beam lithography on a thin silicon nitride membrane [6]. Sometimes a second path, in the form of a small tube, is placed in parallel with the aperture. One wall of the box contains a flexible metalized plastic membrane that can be manipulated by applying potentials to an adjacent fixed electrode. The membrane serves as the oscillator spring and the kinetic energy of superflow in the aperture provides the oscillator inertia [see (1)]. By monitoring the position of this membrane we can detect the superflow through the aperture. The position sensing is done with a DC-SQUID and is similar to sensors used on gravity-wave detectors [10].

${ }^{1}$ Another miniaturized oscillator is described in [5] 


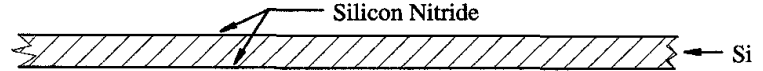

(a)

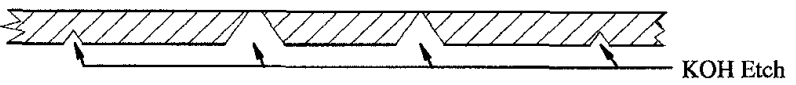

(b)

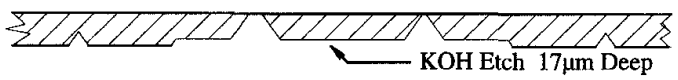

(c)

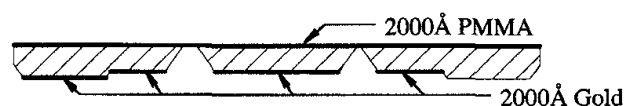

(d)

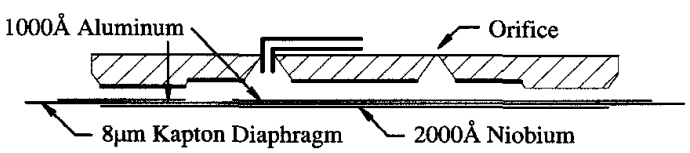

(e)

Fig. 2. Microfabrication and construction steps. See text.

We choose a single hole device with no second flow path to study exclusively the flow through the small aperture. This is useful for experiments aimed at studying the critical velocity and phase slip nucleation in superfluid. When a second path is constructed, we allow for the possibility of quantized circulating superflow to thread the aperture and the fine tube. This configuration is useful for development of a device which is the analog of the superconducting RF-SQUID [3].

\section{COnStruction}

The fabrication of the inner superfluid filled box has three stages and is accomplished at the University of California, Berkeley Microlab. First, the box is micromachined from silicon to form the interior volume and also to create the silicon nitride membrane for the aperture. Second, the aperture is fabricated using electron beam lithography and reactive ion etch (RIE). Finally a flexible Kapton diaphragm is attached over the cavity to complete the inner box. We will not discuss the fabrication of the aperture since it has been discussed in previous publications [6].

The fabrication steps are displayed in Fig. 2. The processing begins with "prime" p-type (100) silicon wafers, nominally $500 \mu \mathrm{m}$ thick and $100 \mathrm{~mm}$ diameter. In principle this wafer can accommodate over 100 individual, 8-mm square dies, each of which is a potential Helmholtz oscillator. We deposit $900 \AA$ of low stress silicon nitride over both sides of the entire wafer [7]. See Fig. 2(a). This material serves two purposes; the film on one side (the back) serves as a mask for the later silicon machining (in $\mathrm{KOH}$ ), and the film on the other side (the front) will contain the aperture.
We then create two square, silicon nitride membranes [8], [9] on the front surface using standard photolithography, plasma etching, and bulk silicon etching in $\mathrm{KOH}$. These membranes are $30 \mu \mathrm{m} \times 30 \mu \mathrm{m}$ and $250 \mu \mathrm{m} \times 250 \mu \mathrm{m}$. At this time we also etch grooves, $200 \mu \mathrm{m}$ deep, around the perimeter of the $8 \mathrm{~mm}$ die, which are used to break apart the wafer without using a dicing saw. See Fig. 2(b). We avoid the dicing saw to keep the wafer clean for the electron beam lithography.

Next, using photolithography, RIE, and $\mathrm{KOH}$, we etch a shallow depression on the back side, $5.2 \mathrm{~mm} \times 5.2 \mathrm{~mm}$ and 17 $\mu \mathrm{m}$ deep. This leaves a raised perimeter $\sim 1.5 \mathrm{~mm}$ wide around the entire die. See Fig. 2(c). This procedure is problematic since during the application of the photoresist, the previously machined pits and grooves cast a "shadow," rendering the photoresist nonuniform. Because of this, the typical yield per wafer is only five to ten devices in the center of the wafer. The procedure to spread the photoresist is to first cover the entire wafer with resist, then spin slowly to throw off most of the resist. The wafer is brought to a stop to let the remaining resist relax to a more uniform thickness for about $5 \mathrm{~s}$. Finally, the wafer is spun up gradually to 3000 RPM for $45 \mathrm{~s}$. This leaves a layer of photoresist of unknown thickness that can be used nevertheless by heavily over exposing and over developing. Since there are no fine features which are exposed at this stage of photolithography, the imprecision of this crude step is inconsequential.

After the etching of the shallow pit with $\mathrm{KOH}$, the wafer is cleaned in piranha ${ }^{2}$ to prepare it for a coating of PMMA electron beam resist. The PMMA is spun on the front surface of the wafer and is baked at $170^{\circ} \mathrm{C}$ for $2 \mathrm{~h}$.

We evaporate $2000 \AA$ of gold over $75 \AA$ of chrome, on the back side of the wafer to serve as an actuating electrodes. ${ }^{3}$ The chrome and gold is evaporated at an angle such that no metal will be deposited on the silicon nitride membranes. See Fig. 2(d). If the gold is deposited on the membrane it will interfere later in the plasma etching process that makes the aperture. Before evaporation we mask one side of each device with a small piece of plastic tape. After the evaporation step the tape is removed. This creates an electrically insulating path for the connection going to the actuating electrode on the Kapton. (See discussion below.)

After the gold deposition, the wafer is broken up into individual devices and is ready for electron beam lithography. ${ }^{4}$

We produce the aperture in the $30 \mu \mathrm{m}$ window following the procedures of [6]. After producing the aperture, the larger silicon nitride membrane is now either sealed with a small dot of epoxy $y^{5}$ to create a single aperture device, or the membrane is punctured and an appropriate tube is epoxied in place to create a two-hole device. See Fig. 2(e). For the device with two holes, the tube was a $250 \mu \mathrm{m}$ o.d. $50 \mu \mathrm{m}$ i.d. copper tube, $4.0 \mathrm{~mm}$ long.

${ }^{2}$ Piranha is a self-heated mixture of sulfuric acid and hydrogen peroxide mixed $5: 1$.

${ }^{3}$ We chose gold as the electrode material since it is an excellent electrical conductor, and with $75 \AA$ chrome evaporated first, it is known to adhere well to silicon.

${ }^{4}$ We use a Jeol 6400 electron microscope configured for lithography.

${ }^{5}$ Stycast 2850 . Stycast epoxies are available from E. V. Roberts \& Associates, 8500 Stellar Dr., Culver City, CA 90232 USA. 


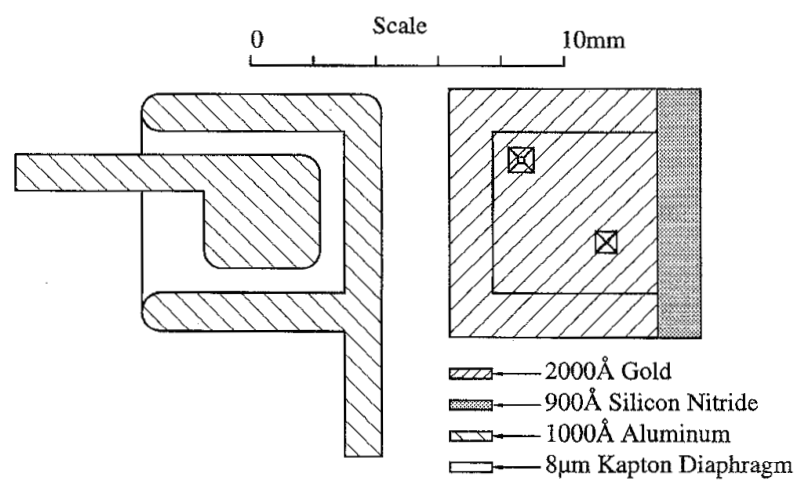

Fig. 3. Aluminum electrode pattern on the Kapton diaphragm and gold electrode pattern on the silicon device.

The inner box of the oscillator is completed by attaching a flexible $8 \mu \mathrm{m}$ thick Kapton diaphragm to the back side. The membrane is prepared with patterned metallic films. On the side which will be internal to the device, $1000 \AA$ of aluminum $^{6}$ is deposited by evaporation using a shadow mask. The electrode pattern is shown in Fig. 3. The electrode which is patterned on the perimeter of the diaphragm makes electrical contact to the gold deposited on the silicon. The electrode in the center of the diaphragm is insulated from the gold on the silicon and exits from the interior space over the nitride covered edge. A parallel plate capacitor is formed by the center electrode on the Kapton and the gold-covered silicon $17 \mu \mathrm{m}$ away. The exterior side of the Kapton diaphragm is coated with $2000 \AA$ of niobium ${ }^{7}$ by sputtering through a shadow mask. This film serves as the superconducting plane for an ultrasensitive squid-based displacement sensor [10]. The finished diaphragm is attached to the silicon body with a very thin film of epoxy. ${ }^{8}$ This joint must be superfluid leak tight and make electrical contact between the gold on the silicon and the aluminum on the Kapton. These two criteria are met without any difficulty.

After the diaphragm is attached, the chip is placed into a brass cell holder shown in Fig. 4. The bottom of the brass holder contains the displacement sensor coil and electrical feedthroughs for the diaphragm electrodes. The displacement sensor input is a pancake coil of superconducting wire. ${ }^{9}$ This coil is 12 turns of wire glued to a brass button with Stycast 1266. This coil is permanently attached to the brass holder and is positioned $<50 \mu \mathrm{m}$ away from the flexible diaphragm. This feature permits one to change the silicon oscillator without remaking and repositioning the displacement sensor coil.

Electrical contact to the aluminum film on the Kapton membrane is made by a pinch contact between an indium coated washer and the cell holder. The soft indium coating prevents ripping the Kapton diaphragm or damaging the 1000 $\AA$ aluminum film.

\footnotetext{
${ }^{6}$ We choose aluminum because it is known to adhere well to Kapton.

${ }^{7}$ We choose niobium since it has a superconducting transition above 4.2 $K$ which allows tests of the displacement sensor in a helium bath. It is also known to adhere well to Kapton.

${ }^{8}$ Stycast 1.266

${ }^{9} 10$ mil o.d. copper clad niobium-titanium wire.
}

The brass cell containing the silicon device is surrounded by a lead box and then a niobium cylinder. These serve as superconducting magnetic shields for the displacement sensor.

The cell is filled through a fine capillary which is then sealed with a hydraulic needle-valve ${ }^{10}$ situated on the refrigerator. This valve thermally and acoustically isolates the superfluid sample. This assembly is attached to a homemade, one-shot evaporation ${ }^{3} \mathrm{He}$ refrigerator which has a base temperature of $0.23 \mathrm{~K}$ and a run time of $\sim 40 \mathrm{~h}$.

The internal cavity formed by the silicon and the Kapton diaphragm has a total volume of $0.72 \mathrm{~mm}^{3}$. The volume from the two pyramidal cavities etched to create the silicon nitride membranes is $0.18 \mathrm{~mm}^{3}$ while the $17 \mu \mathrm{m}$ gap contributes 0.55 $\mathrm{mm}^{3}$. It would be difficult to make this device with significantly smaller volume. This cell has an internal volume 40000 times smaller than previous cells reported from Berkeley [11] and $10 \times$ smaller than the cell reported in [5].

\section{Performance, Sensitivity, And, Noise Immunity}

This system of a submicron aperture and flexible diaphragm has a hydrodynamic resonance whose angular frequency is given by

$$
\omega=\sqrt{\frac{\rho_{s} k a_{o}(1+R)}{\rho_{o}^{2} l_{o} A^{2}}} \frac{\mathrm{rad}}{\mathrm{s}}
$$

where:

$\rho_{s}$ the superfluid density ${ }^{11}\left(0\right.$ at $T=2.17 \mathrm{~K}$ to $145\left(\mathrm{~kg} / \mathrm{m}^{3}\right)$ at $T=0 \mathrm{~K}$ );

$\rho_{o}$ the density of liquid helium $\left[145\left(\mathrm{Kg} / \mathrm{m}^{3}\right)\right]$;

$a_{\circ}$ the area of the aperture (ranging from $7.85 \cdot 10^{-15} \mathrm{~m}^{2}$ to $4.6 \cdot 10^{-13} \mathrm{~m}^{2}$ );

$l_{o}$ the effective hydrodynamic length of the aperture (ranging from $1.7 \cdot 10^{-7} \mathrm{~m}$ to $6.9 \cdot 10^{-7} \mathrm{~m}$ );

$a_{t}$ he area of the small tube $\left(2.0 \cdot 10^{-9} \mathrm{~m}^{2}\right)$;

$l_{t}$ the effective hydrodynamic length of the tube $\left(4.0 \cdot 10^{-3}\right.$ $\mathrm{m}$ );

$A$ the area of the Kapton diaphragm $\left(2.7 \cdot 10^{-5} \mathrm{~m}^{2}\right)$;

$k$ the spring constant of the diaphragm $\left[1.64 \cdot 10^{4}(\mathrm{~N} / \mathrm{m})\right]$; $R=\left(l_{o} / a_{o}\right) \cdot\left(a_{t} / l_{t}\right)(R=7.8$ for two hole and 0 for one hole experiment).

The resonant frequencies range from $40.5-75.2 \mathrm{~Hz}$. The quality factor for a two-hole oscillator spans from $Q=10000$ at $0.25 \mathrm{~K}$ to $Q=4$ at $2.17 \mathrm{~K}$. For temperatures below 1.3 $\mathrm{K}$, the quality follows a $T^{-5}$ dependence. This temperature dependence is consistent with dissipation caused by heat flow between the inner volume of the cell and the outer superfluid reservoir. ${ }^{12}$ The heat flow is driven by the small temperature difference created by the changes in specific entropy. These changes in entropy are caused by the pure superflow through the holes.

\footnotetext{
${ }^{10}$ A description of this hydraulic needle valve will appear in the Proceedings of the XXI International Conference on Low Temperature Physics. This will be published in the Czechoslovak Journal of Physics.

${ }^{11}$ For an introduction to superfluidity see [12]

${ }^{12}$ The calculation of this thermodynamic dissipation will be published by S. Backhaus.
} 


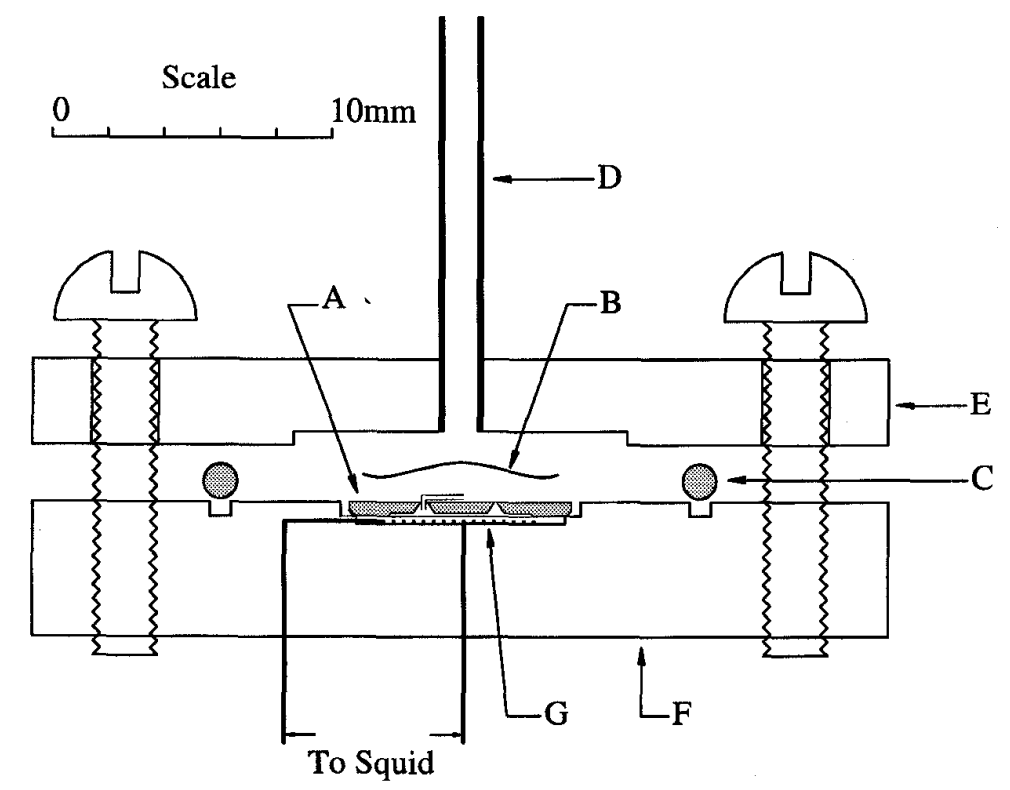

Fig. 4. Device holder: A-superfluid oscillator, B-beryllium-copper spring to hold device against displacement sensor, C--indium o-ring, D-helium filling capillary, E-brass lid, F-brass bottom, and G-flat superconducting coil displacement sensor.

The response of the system is studied by driving the diaphragm at the resonant frequency. The driving force is supplied by applying an oscillating voltage between the gold electrode on the silicon and the aluminum electrode on the diaphragm. A computer is used to find and record the peak amplitude of each half-cycle of oscillation. We also record the response with a lock-in detector.

If one starts with the superfluid oscillator initially at rest and then begins to apply a resonant driving force, the oscillator will respond as a lightly damped simple harmonic oscillator. When the amplitude of oscillation exceeds some critical velocity in the small aperture, a sudden loss in energy between consecutive half-cycles is observed. This discrete dissipation is caused by the nucleation, motion, and annihilation of a single quantized vortex [13]. The changes in the oscillator energy and the diaphragm amplitude after this event are

$$
\begin{aligned}
\Delta E & =\rho_{o} v_{c} a_{s} \kappa \\
\Delta A_{d} & =\frac{\rho_{s}}{\rho_{o}} \cdot \frac{\kappa}{l_{s} \omega} \cdot \frac{a_{s}}{A}
\end{aligned}
$$

where $v_{c}$ is the critical velocity for superflow in the aperture and $\kappa$ is the quantum of circulation. ${ }^{13}$

Fig. 5 shows these quantized dissipation events in a twohole device. In this device $a_{s}=2.0 \cdot 10^{-13} \mathrm{~m}^{2}, R=2.7, v_{c}$ $=0.74 \mathrm{~m} / \mathrm{s}$ thus $\Delta E=2.1 \cdot 10^{-18} \mathrm{~J}$ and $\Delta A_{d}=2.2 \cdot 10^{-12}$ $\mathrm{m}$ for each dissipation event. Each point plotted represents the amplitude of an oscillation half-cycle, with the open circles and closed circles representing the two flow directions. This data is from a superfluid ${ }^{4} \mathrm{He}$ sample mixed with $0.5 \%{ }^{3} \mathrm{He}$. Notice that there are two consecutive dissipation events. This is

\footnotetext{
${ }^{13} \kappa=h / m_{4}$ where $h$ is Planck's constant and $m_{4}$ is the mass of the ${ }^{4} \mathrm{He}$ atom. Numerically, $\kappa=1.0 \cdot 10^{-7}\left(\mathrm{~m}^{2} / \mathrm{s}\right)$.
}

the expected response [14] for a two-hole device with $R>1$. One can see from Fig. 5 that the sensitivity of this device to individual dissipation events is good. Under favorable electronic noise situations, a dissipation event can be resolved to about $10 \%$ with a measurement time of one half-cycle. With this measurement time, the displacement sensitivity is $A_{\min }=3 \cdot 10^{-13} \mathrm{~m}$ and energy sensitivity is $E_{\min }=2 \cdot 10^{-19}$ $\mathrm{J}$ measured at critical velocity.

We also study the response of the two-hole device to a slowly ramping amplitude resonant driving force. A staircase response curve is expected [14], [15]. This response represents the quantization of circulation around a flow path threading the aperture and the small tube. Fig. 6 shows typical response curves for a sample of ${ }^{4} \mathrm{He}$ mixed with $0.1 \%{ }^{3} \mathrm{He}$, from $0.28-0.58 \mathrm{~K}$. The parameters for this cell were: $a_{s}=7.85$. $10^{-15} \mathrm{~m}^{2}, a_{t}=2.0 \cdot 10^{-9} \mathrm{~m}^{2}, l_{t}=4.0 \cdot 10^{-3} \mathrm{~m}$, and $R=7.8$. The light dotted line segment on the curve marked $0.28 \mathrm{~K}$ of Fig. 6 is to guide the eye since data was not taken there.

It is interesting to note that the step response is a consequence of the quantization of circulation around a macroscopic path. This quantization is analogous to the quantization of flux in a superconductor. In the two-hole device the circulation path has a total path length of $8.5 \mathrm{~mm}$ and an enclosed area of 1.9 $\mathrm{mm}^{2}$.

We find the susceptibility to acoustic noise to be much lower in comparison to other cells experimented with at Berkeley. When undriven, the oscillator amplitude always decayed below the white-noise floor of our squid detector, $\approx 2$. $10^{-5}\left(\Phi_{\circ} / \sqrt{\mathrm{Hz}}\right)$. This places an upper bound on the spurious drive amplitude of $<10^{-1} \cdot \Delta A_{d}$. This is a considerable improvement in comparison to our previous larger oscillators which, despite efforts to eliminate spurious driving forces, were always found to be driven to an amplitude comparable to $\Delta A_{d}$. We find no measurable effect from turning on 


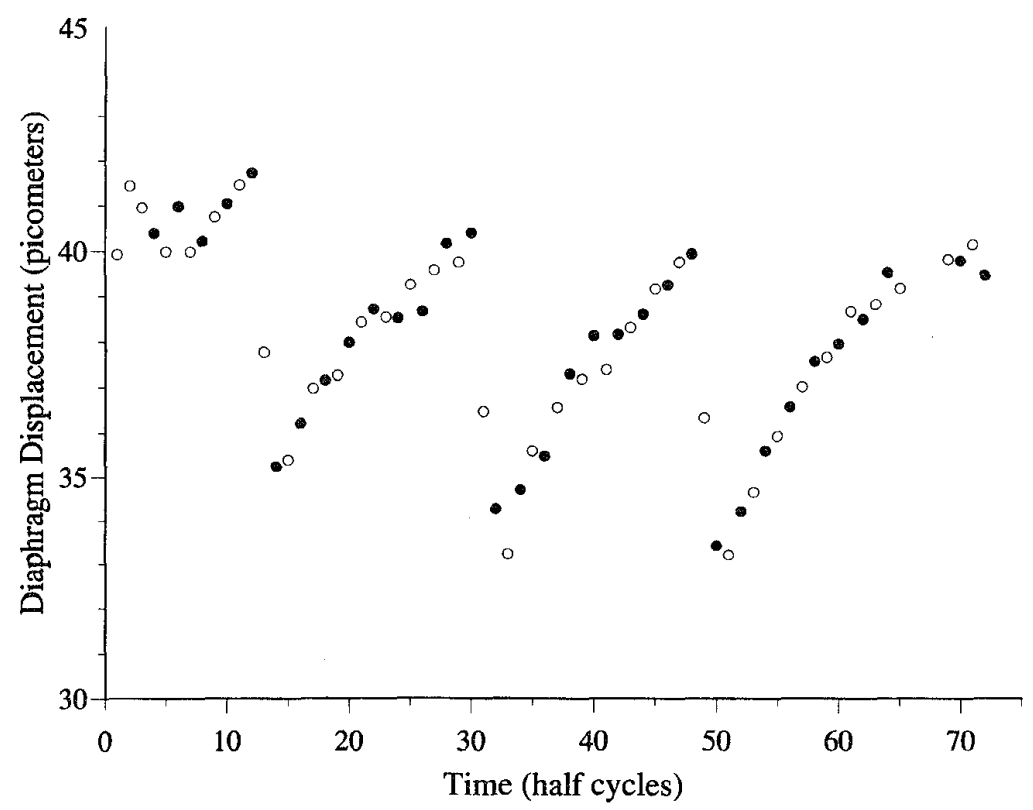

Fig. 5. Oscillator amplitude versus time for a fixed drive. The points represent peak amplitude of a half-cycle and time is measured in oscillator half-cycles. The different symbols represent the two flow directions. This is a ${ }^{4} \mathrm{He}$ sample mixed with $0.5 \%{ }^{3} \mathrm{He}$. The data was taken with a two-hole configuration at $T=0.25 \mathrm{~K}$.

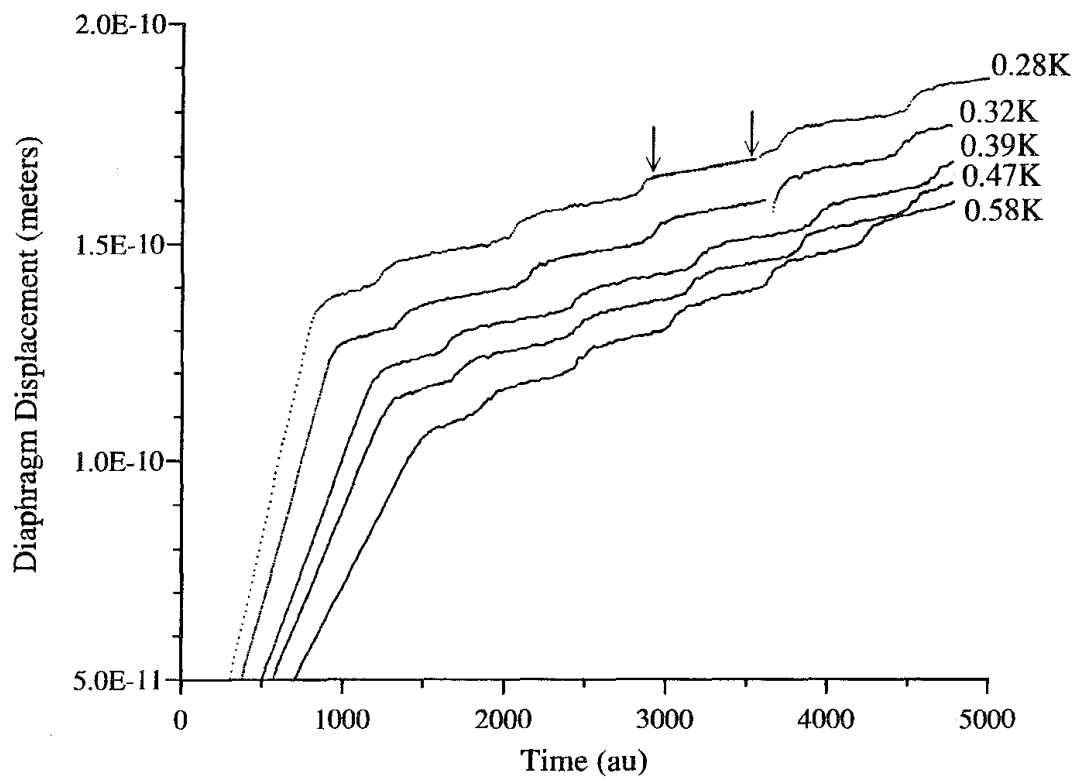

Fig. 6. Typical staircase response in a two-hole resonator. Oscillator amplitude vetsus time/drive is recorded with a lock-in detector. This data is from a ${ }^{4} \mathrm{He}$ sample mixed with $0.1 \%{ }^{3} \mathrm{He}, T=0.29 \mathrm{~K}$. See text for cell parameters.

or off a large mechanical pump which holds the helium bath at low pressure. We find no measurable effect on the critical velocity or on the staircase response with noise in the surrounding laboratory from people moving and talking, electronic equipment, or room fluorescent lighting "hum." This is a dramatic improvement in comparison to large volume oscillators where suppression of the critical velocity or sudden decays of the oscillator amplitude where common if the acoustic environment is not ideal. We have never observed a sudden amplitude decay with the one-hole device. In the twohole device, sudden amplitude decay is observed only with severe acoustic and vibrational drive, such as the slamming of a nearby door. In Fig. 6, on the trace marked $0.32 \mathrm{~K}$ one can see the effect of a slammed door. The oscillator amplitude decays and recovers quickly, with no effect on the next steps.

Beyond ambient acoustic noise, we have measured the sensitivity of this device to applied acoustic drive generated by an external loudspeaker. These experiments were performed on 
the one-hole configuration. We applied three spectra of sound: white-noise, $100 \mathrm{~Hz}$, and the oscillator resonant frequency of $40.5 \mathrm{~Hz}$. The oscillator was found to be most susceptible to sound at its resonant frequency. We were able to drive the oscillator to its critical amplitude and observe high quality phase-slippage with an applied ambient sound level of 86 $\mathrm{dB}$.

In comparison to the device used by Avenel and Varoquaux, we have less sensitivity for detecting the phase slip dissipation events. This is simply because of the extremely low spring constant of their Kapton diaphragm, $\sim 300 \mathrm{Nt} / \mathrm{m}$ (versus our $16400 \mathrm{Nt} / \mathrm{m}$ which is determined by the relative thermal contraction between the Kapton and the silicon [16]). This low spring constant generates a lower resonant frequency (1) and consequently a larger diaphragm displacement (2) for the phase slips. This places the oscillator resonance into a lower frequency range, 4-12 Hz for the Avenel and Varoquaux cell, however, that is increasingly difficult to isolate from external vibrations. For the silicon oscillator, it is possible to improve the displacement sensitivity by using a higher turndensity sensing coil. We have recently made this improvement and have demonstrated a displacement sensitivity increase of a factor of $\sim 3$. Because the vibrational environment plays such a crucial role in the performance of these devices, it is advantageous to improve the displacement sensor and operate at higher frequencies. It is also desirable for a future gyroscope to operate at the highest frequency possible. As with a dissipative superconducting RF-SQUID, the noise floor is ultimately limited by the thermal distribution of the phase slips. For a given operating temperature, the noise is then reduced by maximizing the number of slips per time [3].

\section{CONClusions AND Future DiRECTIONS}

We have developed a high performance miniaturized superfluid oscillator of the type successfully demonstrated by Avenel and Varoquaux. This device shows dramatically improved performance compared to larger cells experimented with at Berkeley. We believe that the miniaturization of the cell volume, and thereby maximizing the acoustic resonant frequencies, is responsible for this improvement and is an important design parameter for future devices. By utilizing microfabrication techniques and batch processing we have been able to quickly test two device configurations and are currently in the process of testing more of these devices. Device insertion time into the apparatus is minimized by integrating the displacement sensor to the brass cell holder. This is a significant design advantage to aid in oscillator development and optimization. The sensitivity to quantized dissipation events is excellent, and when it is configured with two holes, the device shows a repeatable, high quality staircase response.

The performance of these devices is very encouraging. We are currently testing additional two-hole devices in ${ }^{4} \mathrm{He}$ with the intention of developing a superfluid gyroscope [3]. We have designed and fabricated a new cell which has a large calculated sensitivity to rotation. ${ }^{14}$ The device is $15 \mathrm{~mm}$ square and is constructed in a similar fashion to the original device described here. This device should have the sensitivity to resolve the Earth's rotation with a $\mathrm{S} / \mathrm{N} \sim 100$.

We are also making preparations for experiments with superfluid ${ }^{3} \mathrm{He}$ on a sub-millikelvin refrigerator. For superfluid ${ }^{3} \mathrm{He}$, the apertures are of the size of the coherence length and have been found to behave as Josephson junctions [15]. We anticipate that these silicon-based superfluid oscillators will also yield information about Josephson effects in this superfluid and possible new devices based on these effects. Specifically, a device analogous to the superconducting DCSQUID is an exciting possibility [3].

\section{ACKNOWLEDGMENT}

The authors would like to acknowledge the many helpful discussions with Y. Muhkarsky, S. Backhaus, and A. Rimberg. They would also like to acknowledge the technical assistance from the Berkeley Sensors and Actuators Group and the staff of the Berkeley Microlab, especially D. Hebert and D. Hebert.

\section{REFERENCES}

[1] O. Avenel and E. Varoquaux, "Observation of singly quantized dissipation events obeying the Josephson frequency relation in the critical flow of superfluid ${ }^{4} \mathrm{He}$ through an aperture," Phys. Rev. Lett., vol. 55, no. 24, pp. 2704-2707, Dec. 1985.

[2] J. Steinhauer, K. Schwab, Y. Mukharsky, J. C. Davis, and R. E. Packard, "Vortex nucleation in superfluid ${ }^{4}$ He," Phys. Rev. Lett., vol. 74, no. 25, pp. 5056-5059, June 1995.

[3] R. E. Packard and S. Vitale, "Principals of superfluid-helium gyroscopes," Phys. Rev. B, vol. 46, no. 6, pp. 3540-3549, Aug. 1992.

[4] O. Avenel, G. G. Ihas, and E. Varoquaux, "The nucleation of vortices in superfluid ${ }^{4}$ He: Answers and questions," J. Low Temp. Phys., vol. 93 no. 5/6, pp. 1031-1057, Dec. 1993.

[5] O. Avenel and E. Varoquaux, "A miniaturized low frequency Helmholtz resonator for the study of superfluid flow through an aperture," in Proc. XIth Int. Cryogenic Eng. Conf., 1986, pp. 587-591.

[6] A. Amar, Y. Sasaki, R. Lozes, J. C. Davis, and R. E. Packard, "Fabrication of submicron apertures in thin membranes of silicon nitride," J. Vac. Sci. Tech. B, vol. 11, no. 2, pp. 259-262, Mar./Apr. 1993.

[7] M. Sekimoto, H. Yoshihara, and T. Ohkubo, "Silicon nitride singlelayer X-ray mask," J. Vac. Sci. Tech., vol. 21, no. 4, pp. 1017-1021, Nov./Dec. 1982

[8] R. M. Finne and D. K. Klein, "A water-amine-complexing agent system for etching silicon," J. Electrochem. Soc., vol. 114, no. 9, pp. 965-970, Sept. 1967.

[9] A. Reisman et al., "The controlled etching of silicon in catalyzed ethylenediamine-pyrocatechol-water solution," J. Electrochem. Soc., vol. 126, no. 8, pp. 1406-1415, Aug. 1979.

[10] H. J. Paik, "Superconducting tunable-diaphragm transducer for sensitive acceleration measurements," $J$. Appl. Phys., vol. 47, no. 3, pp. 1168-1178, Mar. 1976.

[11] A. Amar, Ph.D. dissertation, University of California, Berkeley, 1992

[12] D. R. Tilley and J. Tilley, Superfluidity and Superconductivity, 2nd ed. Bristol: Adam Hilger Ltd., 1986.

[13] P. W. Anderson, "Considerations on the flow of superfluid helium," Rev Mod. Phys., vol. 38, no. 2, pp. 298-310, Apr. 1966.

[14] B. P. Beecken and W. Zimmermann, Jr., "Search for an ac Josephson effect in superfluid ${ }^{4} \mathrm{He}$ using a low-frequency acoustic resonator," Phys. Rev. B. vol. 35, no, 1, pp. 74-88, Jan. 1987.

[15] O. Avenel and E. Varoquaux, "Josephson effect and quantum phase slippage in superfluids," Phys. Rev. Lett., vol. 60 , no. 5, pp. 416-419, Feb. 1988.

\footnotetext{
${ }^{14} \mathrm{~A}$ brief description of this gyroscope design will appear in the Proceedings of the XXI International Conference on Low Temperature Physics This will be published in the Czechoslovak Journal of Physics.
} 
[16] J. M. Roe and R. Simha, "Thermal expansivity and relaxational behavior of amorphous polymers at low temperatures," Intern. J. Polymeric Mater., vol. 3, pp. 193-227, 1974.

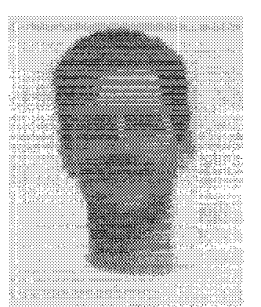

Keith Schwab was born in St. Louis, MO, in 1986. He received the B.A. degree in physics from the University of Chicago, IL, in 1990. He is expected to complete the Ph.D. degree from the University of California, Berkeley, in the fall of 1996.

He was awarded the 1996 Sherman Fairchild Fellowship from the California Institute of Technology, Pasadena, where he will start as a Post Doctoral Researcher in the fall of 1996.

J. Steinhauer, photograph and biography not available at the time of publication.
J. C. Davis, photograph and biography not available at the time of publication.

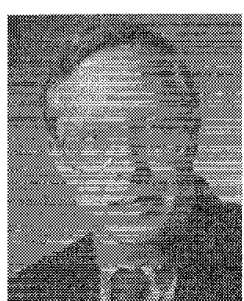

Richard E. Packard is a Physics Professor at the University of California at Berkeley. He specializes in the study of superfluid helium, often utilizing microfabrication technology. 